\section{Reduction of resting state network segregation is linked to} disorders of consciousness

Jorge Rudas ${ }^{a}$, Darwin Martínez ${ }^{a, d}$, Javier Guaje ${ }^{a}$, Athena Demertzi ${ }^{b}$, Lizette Heine ${ }^{b}$, Luaba Tshibanda $^{b}$, Andrea Sodd ${ }^{c}$, Steven Laureys ${ }^{b}$, Francisco Gómez ${ }^{d}$

${ }^{a}$ Computer Science Department, Universidad Nacional de Colombia, Colombia ${ }^{b}$ Cyclotron Research Center, University of Liége, Belgium ${ }^{c}$ Physics and Astronomy Department, Wester

University, Canada ${ }^{d}$ Computer Science Department, Universidad Central, Colombia

\section{ABSTRACT}

Recent evidence suggests that healthy brain is organized on large-scale in regions spatially distant and partially temporally synchronized. These regions commonly are called Resting State Networks (RSNs). Many RSNs has been identified in multiples spatial scales in healthy subjects and their interactions has been used to define the fanctional network concetivity (FNC). The main idea in FNC is that the dynamic shown in the interactions among RSNs in control subjects, can change in pathological and pharmacological conditions. However, this hypothesis assumes that functional structure of healthy brain, remains in other brain states or conditions. In this work, we proposed a novel methodology in order to find of RSNs set, that maximize two modularity measures (Kapur and Otsu measures). Our results sugrest that the brain segregation level, may be linked to consciousness level.

Keywords: Community, disorders of consciousness, NSGA II, resting state networks, segregation.

\section{INTRODUCTION}

Recent studies on functional magnetic resonance imaging (fMRI) suggest that healthy brain in resting state is organized into large-scale of resting state networks (RSNs). ${ }^{1}$ The existence of at least ten of these RSNs (default mode network (DMN), executive control network left (ECL), executive control network right (ECR), saliency, sensorimotor, auditory, cerebellum and three visual networks medial, lateral and occipital) have been consistently reported in healthy subjects. ${ }^{2}$ Each RSN encompasses a set of spatial regions with a common functional behavio or time-course, and corresponds to a functional description of a high level brain system of cognitive/sensorial relevance. ${ }^{3}$ The RSN time courses, in turn, can be used to construct a large scale functional connectome that (FNC) ${ }^{4}$ In this approach, the degree of interaction among the RSNs time-courses is quantified, resulting in very-large-scale functional network whose nodes are RSNs. In contrast, to other functional networks, which are conformed by smaller brain areas, the FNC model of connectome aims to characterize high level interactions among complete brain functional systems. ${ }^{5,6}$

Several brain pathological conditions including disorders of consciousness, dementia, Alzheimer disease, among others, have been studied by using the RSN approach. ${ }^{2,5-10}$ Most studies mainly focus on changes in the intrinsic connectivity of one RSN, typically, the DMN. Nevertheless, there is evidence that suggests that rather than one, multiple RSNs maybe affected during pathological conditions. For instance, patients with disCR ancons alousness may show alterations of the intrinsic connectivity on at least four RSNs: ${ }^{2}$ DNN, ECL, ECR and auditory. Recent studies have also linked changes in the interactions among these functional systems to - ECR and visual medial - salience have been recently related to conditions of severe impairment in patients with Further author information: (Send correspondence to Francisco Gómez.) Francisco Gómez:: E-mail: fagomezj@gmail.com, Bogotá, Colombia, Central University, Lino Pombo Bulding, Second
Floor, Race. 5 N. 21-38

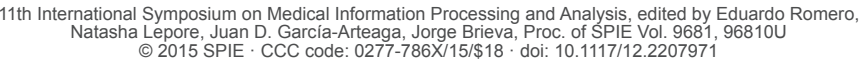

DOCs. ${ }^{5}$ This panorama suggests that pathological brain states may be associated to changes in individual high level functional systems, but also to alterations in the communication among them.

The brain is a highly complex functional structure, with a densely connected network that balances regional segregation with integration throughout multiple spatio-temporal scales. ${ }^{12,13}$ The brain segregation, observed for instance in resting state sponten the cognitive or sensory processing tasks. In other hand, the brain integration, observed for example in the ric club topology that supports efficient brain communication, ${ }^{14}$ suggest that even if the brain is organized into segregated systems, these systems are still interacting among them. ${ }^{15}$ The segregation and integration do no operate as independent phenomenas, rather both properties seems to be required to provide a coordinated activation. It have been suggested that perturbations in the unbalance between segregation and integration may be associated with pathological brain conditions. ${ }^{17}$ For instance, recent computational models sugges that too much integration among brain systems, which are normally segregated, may facilitate the abnorm

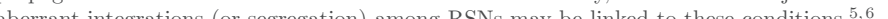

Recently, it have been suggested that segregation mechanisms are strongly supported by a particular grap structure called community. A community refers to a sets of brain regions that are strongly related amon evidence suggests that community structures are in the very base of the structural segregation mechanisms. ${ }^{17}$ Actually, there are not methods to find the communities among brain RSNs by using only the raw data. without using graph theory and any prior information. In this sense, we propose a novel strategy to find community structures that does not require any prior knowledge about the expected number of communities, an also overcomes the limitations of the traditional functional graph representation. The proposed method is based in an multi-objetive optimization strategy that aims to maximize a novel modularity criterion based on clustering measurements. More specifically, we aim to find the graph partition that better balance a Kapur and the Otsu criterion, ${ }^{21}$ two widely knowledge segmentation criteria commonly used by the image processing community. Ou aim is to find clusters maximally informative and minimally varlant intra-cluster and simultaneously, maximally variant extra-clusters. By using this combination we account for both integration and segregation properties,
The proposed method was used to study the segregation Tugest a brealdown in the segregation of high level functional networks for patients with $\mathrm{DOCs}$ when compared to healthy controls.

1.1 Previous work

In the recent years, different methods have been proposed to extract communities out of functional and structural brain data. These methods can be categorized in: 1) graph based, which corresponds to methods assume an intermediate graph representation of the brain connectivity. The main idea of these approaches to find subgraphs directly from the graph by using, for instance, graph based clustering methods or by characterizing the statistical properties commonly observed for the communities in a network, examples of this category include the min-cut, GirvanNewman methods and clique based methods. ${ }^{22} 2$ 2) modularity maximization, which are methods that
aim to find the graph partition with a maximal modularity. Modularity refers to a measure of the quality of a aim to find the graph partition with a maximal modularity. Modularity refers to a measure of the quality of a particular partition of a network into communities, and can be defined as the fraction of the edges that fall within

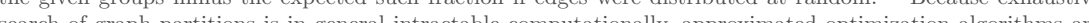
commonly used to find the optimal partition.20 3) clustering the idea of these methods is to find directly the communities as clusters, an example of these methods include hierachical clustering and independent conponent analysis. ${ }^{24}$ Graph based methods are based on an intermediate graph representation of the functional interaction to compute the communities. These graph representations are critically supported on binary relationships between brain regions that may oversimplify the complex functional relationships, which may appear as the result of interactions of more than two regions. ${ }^{20}$ Clustering methods do not assume any bina ip. However, they require prior knowledge about the expected number of clusters. Modularity maximization methods do not number of communities, but are also strongly supported on a graph representations of brai connectivity. 


\section{MATERIALS AND METHODS}

Figure 1 illustrates the proposed approach. First, the data are preprocessed (Section 2.2). Following, the signal is decomposed into functional sources by using spatial Independent Component Analysis (Section 2.2.1). Later, Finally, a multiobjetive optimization method is used to find the partition that provides the better balnee between an Otsu and Kappur criterion (Section 2.3).

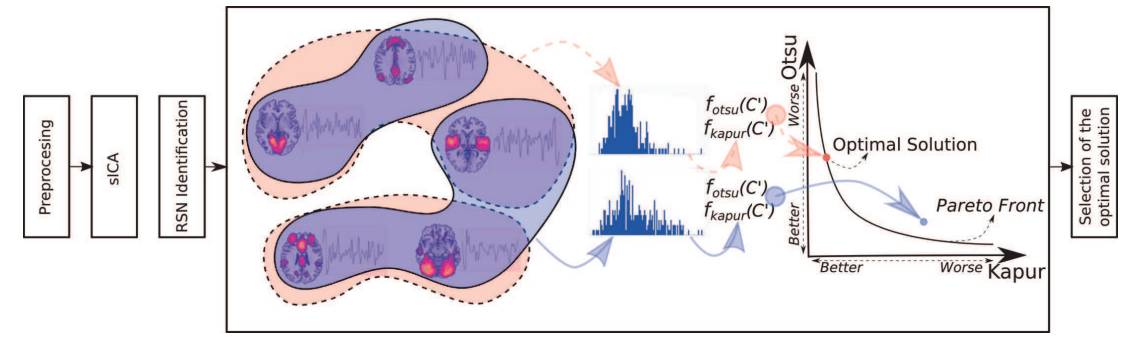

Figure 1. Quantification of segregation approach pipeline. Firstly, the data are preprocessed. Subsequently, the fMR signal is decomposed into functional components by using sICA. Later, a RSN identification procedure is applied to identify the sources corresponding to a set of RSNs. Additionally, a multi-objetive optimization method is used to find the partition that provides the better balance between an Otsu and Kappur criterion. Finally, a selection of the optimal solutio
level.

\subsection{Data acquisition}

Data from 76 subjects were used for this study: 27 healthy controls (14 women, mean age $47 \pm 16$ years), 24 patients in minimal conscious state and 25 with vegetative state/unresponsive wakefulness syndrome (20 women, mean age $50 \pm 18$ years). All patients were clinically examined using the Fench version of the Coma Recovery Sc legal surrogates of the patients. For each subject, fMRI resting data were accuired in a $3 \mathrm{~T}$ scanner (Siemens
or medical Solution in Erlangen, Germany). Three hundred fMRI volumes multislice $T 2^{*}$-weighted functional medical Solution in Erlangen, Germany). Three hundred fMRI volumes multislice $T 2^{*}$-weighted functional
images were captured (32 slices; voxel size: $3 \times 3 \times 3 \mathrm{~mm}^{3}$; matrix size 64 ; repetion time $=2000 \mathrm{~ms}$; echo time $=30 \mathrm{~ms}$; flip angle $=78$; field of view $=192 \mathrm{~mm}^{2}$ ). An structural T1 image was also acquired for anatomical reference. For the resting state acquisition, patients were instructed to close their eyes, relax without falling asleep and refrain from any structured thinking (e.g., counting, singing etc.).

\subsection{Preprocessin}

fMRI data was processed using SPM8*. Preprocessing includes: realignment, coregistration of functional onto structural data, segmentation of structural data, normalization into MNI space and spatial smoothing with a .

\subsubsection{Spatial Independent Component Analysis}

The first step for the RSN identification was the fMRI signal decomposition into sources of neuronal/physiological origin. For this task, we used ICA, which aims to decompose the signal into a set of statistically independent components (ICs) of brain activity. In standard ICA, one considers the mixture as linear and the sources as statistically mutually independent and non Gaussian. ${ }^{26}$ In the fMRI data the spatial dimension is much greater
than temporal one, then, we used spatial ICA (sICA), which decompose the signal into maximally independent

*http:www.fil.ion.ucl.ac.uk/spm

thttp://cibsr.stanford.edu/tools/ArtRepair/ArtRepair.htm spatial maps. ${ }^{27}$ In sICA each spatial map (source) have an associated time course, which corresponds to the Wh this con $t \times v$ data matrix $X$, where $t$ is the number of time points in the time course for fMRI data and $v$ is the number of voxels in the volumes. The temporal behavior for each component is summarized in the matrix $A$ (wit dimensions $t \times k, t$ times point in the record for each source, and $k$ components) and the structure spatial for

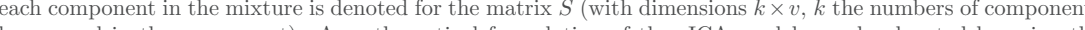
by $v$ voxel in the component). A mathematical formulation of the sICA model, can be denoted by using the

$$
\underset{t \times v}{X}=\underset{t \times k}{A} \times \underset{k \times v}{S}
$$
The RSNs time courses obtained with sICA were subsequently used for all the FNC computations. For the sICA decomposition 30 components $(k=30)$ were used, this selection was performed based on previous work
that have shown that this number of components is enough to characterize the different RSNs both for healthy controls and patients with DOC. The infomax algorithm as implemented in GroupICA toolbox was used to perform the decomposition ${ }^{2}$

2.2.2 RSNs Identification

After the ICA decomposition, the different RSNs were identified at individual level. The common approach for this task is the group level identification. In this method, the fMRI data of whole population is concatenate along the temporal dimension. Later, sICA is applied to identify the sources of brain activity at the group level Following, each RSN is manually identified. Finally, individual time courses are extracted for each RSN by applying a brains, this comlition may be not wat $2^{2}$.

In this work, we used an alternative approach that aims identifying each RSN directly from the single subject sICA decomposition. In particular, we ran a single subject sICA, and then, the set of ICs that maximize the homogenous populations, as the herein studied, and can be used directly for individual assessment of subject in clinical applications. After the RSN spatial map identification, a machine learning based labeling method was applied to discriminate between IC of "neuronal" or "artifactual" origin. In particular, a binary classificatio method based on support vector machines and an spatio-temporal feature vector for description each IC was used. ${ }^{2}$

\subsection{Segregation method}

For the segregation method we propose to find the partition of the RSNs set that maximizes a modularity (segregation) measurent. For this, two measures that quantify the level of segregation of a specific partitio of the RSNs were proposed. The first one aims to quantify the level of information of each community by using a similar idea to the Kapur criterion. The second one considers the combination between both inter-community and intra-commtly problem was solved to find the optimal Pareto frontier based in the two modularity measurements.

\subsubsection{Kapur Criterion}

First, let $C=\left(c_{1}, c_{2}, \ldots, c_{n}\right)$ a vector that assigns each RSN to a particular community. 10 networks were used with the following order: auditory - 1 , cerebellum -2 , DMN -3 , ECL -4 , ECR -5 , saliency - 6 , sensorimoto -7 , visual networks medial -8 , lateral -9 and occipital -10 . The number of communities is noted by $k$ and ranges between $1 \leq k \leq n$, therefore $c_{i} \in\{1,2, \ldots, k\}$. The proposed measurement aims to maximize the sun of entropies for each community, similarly to the Kapur's method, ${ }^{28}$ as follows:

$$
f_{\text {Kapur }}(C)=H_{1}+H_{2}+\ldots+H_{k}
$$

Proc. of SPIE Vol. 9681 96810U-3
Proc. of SPIE Vol. 9681 96810U-4

Downloaded From: http://proceedings.spiedigitallibrary.org/ on 01/22/2016 Terms of Use: http://spiedigitallibrary.org/ss/TermsOfUse.asp 
where $H_{j}$ corresponds to the Shannon entropy of the set composed by the concatenation of all the time-courses of components $c_{i}$ that are assigned to the $k$-th community, i.e, the set composed by all the samples of the time courses of the components in $T_{j}$, with $T_{j}=\left\{i\right.$, such that $c_{i}$ is equal to $\left.j\right\}$. More specifically:

$$
H_{j}=-\sum_{i} p_{i}^{j} \ln p_{i}^{j}
$$

where $p_{i}^{j}$ is the probability occurrence of intensity $i$ in the time courses of all the components inside $T_{j}$. Note that (1) aims to quantify the amount of information among each community. High values for this function can

\subsubsection{Otsu Criterion}

The Otsu's method ${ }^{29}$ is based on the discriminant analysis. Their main idea is to maximize the inter-cluster variance, and simultaneously, to minimize the intra-cluster variance, as follows:

$$
\begin{array}{r}
f_{\text {Otsu }}(C)=\left(u_{1}-u_{2}\right)^{2}+ \\
\left(u_{1}-u_{3}\right)^{2}+\ldots+\left(u_{1}-u_{k}\right)^{2}+ \\
\left(u_{2}-u_{3}\right)^{2}+\left(u_{2}-u_{4}\right)^{2}+\ldots+ \\
\left(u_{2}-u_{k}\right)^{2}+\ldots+\left(u_{k-1}-u_{k}\right)^{2}+
\end{array}
$$

where $u_{j}=\sum_{i} i p_{i}^{j}$

2.3.3 Multiobjetive optimization

Both criteria (1) and (3) are explicitly measurements of modularity-segregation for the FCN network. The Kapur's criterion search the partition that is maximally informative, and Otsu's criterion look for the minimally variant segmentation inside each community and simultaneously, the maximally variant segmentation between communities. However, even if both criteria aims to measure the same network property they differ in their taneously both criteria. This search problem can be solved by using a multi-objetive optimization formulation, as follows:

$$
C^{*}=\operatorname{argmax}_{C}\left(f_{\text {Kapur }}(C), f_{\text {Otsu }}(C)\right)
$$

In this formulation commonly there is no a solution that maximizes both objective functions simultaneously. Therefore, the solution is selected from the Pareto front, i.e., the locus of solutions that cannot be improved in any of the objectives without degrading at least one of the other objectives. ${ }^{30}$

\subsection{Optimization Algorithm}

The exhaustive search of the solution among the complete set of possible RSNs segmentation is computationally intractable. Therefore, we used an heuristics algorithm to approximate the solution. Specifically, we used the fast elitist multi-objetive genetic algorithm (NSGA-II). pareto-optimal solutions, which are those solutions in the set which do not dominate each other, i.e., neither of

\subsubsection{Chromosome representation}

In the NSGA-II approach the possible solutions are codified as chromosomes consisting of $n$ genes, each one organized in a vector structure. In this case, we used $C$ as the chromosome codifying a possible solution.
2.4.2 Optimization parameters

For the NSGA-II optimization we used a multi-objective genetic algorithm with simulated binary crossover (SBX) and polynomial mutation operators implementation ${ }^{8}$. For this experimentation the initial set population wa and reproducibility eriteria. For the others parameters, the default values in the implementation were used.

2.4.3 Optimal solution selection

First, the NSGA-II was used at the subject level to find a non-dominated, as a result 70 feasible solutions were found. Then, a matrix $S_{p} \in R^{70 \times n}$ was constructed by using each feasible solution as row. To find a single solution the mode over the values of each column of $S_{p}$ were computed. This process resulted in a vector $s_{p}$ which represents the solution for a single run. Because NSGA-Il is a non-deterministic algorithm each run can result in a different solution. In order to find an stable solution per-subject, we repeated the optimization 20 times for each subject. Then a similar procedure was used to find an stable solution per-subject, i.e., a matrix the modes over the column of $S_{r}$ were used again to reduce $S_{r}$ to a single solution $s_{r}$ Finally, to fint the solution at the population level a matrix $S_{s} \in R^{q \times n}$ was constructed by using the solutions for the $q$ subject in a determined population. Then the mode was used again over the columns of $S_{\text {s }}$ to find the solution at the population level. An illustration of this procedure is shown in figure 2.

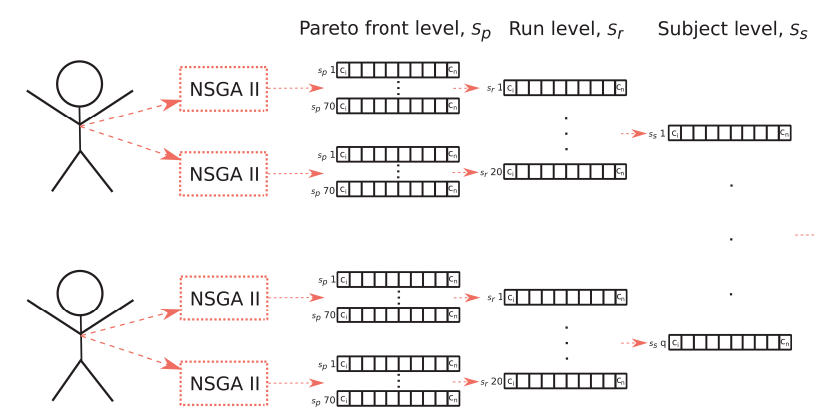

Population level

\section{iD}

Figure 2. Optimal solutions selection flow. Four level of optimal solution selection was applied. In the first level, the mode solution into Pareto front is obtained by one subject in a particular experimental replica, later, the mode solution is anew calculated, this structure is a characteristic solutions at population level.

\section{RESULTS AND DISCUSSIONS}

Figure 3 shows the communities obtained at the population level for the healthy control and the DOC (VS/UWS and MCS) populations by using the procedure described in 2.4.3. As observed, in controls two visual systems (medial and lateral) are grouped into a single community and visual occipital emerge as an independent community. In contrast, in the DOC population these three visual systems conform a single community, suggesting a reduction in the segregation level. A similar result is observed for ECR and ECL that constitute independent communities for healthy controls and conform a single connunity for DOC. Evidence associated with the reduction in the segregation level between $\mathrm{ECR}$ and $\mathrm{ECL}$ in $\mathrm{DOC}$ condition, had already been reported. ${ }^{5}$ lnterestingly, this two two mechanisms that posity $\frac{{ }^{8} \text { https: //atlas.genetics.kcl.ac.uk/ rschulz/ }}{}$ 
Specifically, the executive systems (ECL and ECR) are thought to be involved in handling novel situations, on current task goals as well as responding to salient new information or alerting stimuli in the environment. ${ }^{33}$

A reduction of segregation level is associated with decreased specialization in brain function. ${ }^{34}$ In this context, segregation level is measured through number of communities emerging. Recent evidence, suggests that some brain state or brain pathological conditions may be characterized by alteration in brain segregation level. ${ }^{35-37}$ interactions, can be an oversimplifiction inadequate. In our approach, not is necessary this simplification of problem, because, our methodology is based only on the raw data.
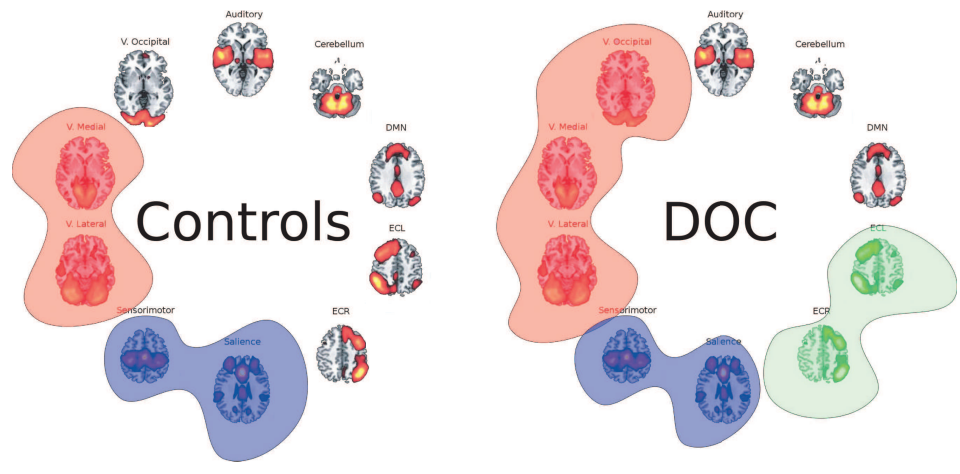

Figure 3. Communities at the populations level. Left communities obtained for healthy controls, and right communities obtained for DOC population (VS/UWS and MCS)

In our results, the number of communities in the DOC population decrease compared to healthy controls

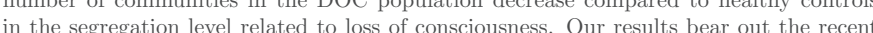
evidence suggesting that the brain integration and brain segregation must coexist in the best balance for an suitable brain functions. ${ }^{35}$ All RSNs reported in the literature, have been consistently and reproducibility identified in healthy subjects, ${ }^{2}$ but, any a Our results suggest, that some RSNs can not exist as segregated entities in DOC condition. Therefore, models as FNC could not be appropriate to characterize pathological conditions as DOC.

\section{CONCLUSIONS}

In this work, we proposed a novel method to find the communities in resting state. Our approach is based in a multi-objective optimization problem that considers two mutually exclusive criteria of modularity-segregation. Our results suggests that the proposed method can find highly the structural conformation of the networks into communities without using any prior information. Our results suggests that the segregation levels in DOC conditions is reduced when compared to control subjects. These results suggest that the segregation level maybe

\section{ACKNOWLEDGMENTS}

This work was supported by the projects Plattorm and Architecture for the representation and data analytics of Páramo leaves morhpology (PARANO) and the Cluster in Convergent Technologies from Universidad Central (FNRS), the European Commission, the James
McDonnell Foundation, the European Space Agency, Mind Science Foundation, the French Speaking Community Concerted Research Action, the Belgian interuniversity attraction pole, the Public Utility Foundation "Universit

\section{REFERENCES}

M. Fox and M. Raichle, "Spontaneous fluctuations in brain activity observed with functional magnetic resonance imaging." Nat Rev Neurosci 8, p. 700711, 2007.

2. A. Demertzi, F. Gómez, J Crone, A Vanhaudenhuyse, L. Tshibanda, Q. Noirhomme, M. Thonnard, V. Charland-Verville, M. Kirsch, S. Laureys, and A. Soddu "Multiple fmri system-level baseline connectivity is disrupted in patients with consciousness alterations," Cortex 52(0), pp. 35 - 46, 2014.

3. J. S. Damoiseaux, S. A. R. B. Rombouts, F. Barkhof, P. Scheltens, C. J. Stam, S. M. Smith, and C. F. Beckmann, "Consistent resting-state networks across healthy subjects," Proceedings of the National Academ of Sciences 103(37), pp. 13848-13853, 2000.

4. M. Jafri, G. Pearlson, M. Stevens, and V. Calhoun, "A method for functional network connectivity among spatially independent resting-state components in schizophrenia," Neurolmage 39(4), pp. 1666 - 1681, 2008 J. Rudas, J. Guaje, A. Demertzi, L. Heine, L. Tshibanda, A. Soddu, S. Laureys, and F. Gomez, "A method (6.

6. J. Rudas, J. Guaje, A. Demertzi, L. Heine, L. Tshibanda, A. Soddu, S. Laureys, and F. Gómez, "Dynamic X. Liu, B. D. Ward J. R. Binder S. J. Li, and A. G. Hudetz "Scale-free functional connectivity of the

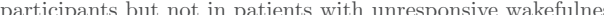
syndrome," PLoS ONE 9, p. e92182, 032014.

8. S. Spadone, S. Della Penna, C. Sestieri, V. Betti, A. Tosoni, M. G. Perrucci, G. L. Romani, and M. Corbetta, "Dynamic reorganization of human resting-state networks during visuospatial attention," Proceedings of the National Academy of Sciences 112(26), pp. 8112-8117, 2015.

9. C. Jungho, H. Jung-Min, J. J. Hang, W. S. Sang, N. Duk, and L. Jong-Min, "Assessment of functiona characteristics of amnestic mild cognitive impairment and alzheimers disease using various methods

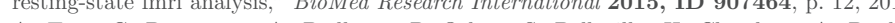

10. A. Tam, C. Dansereau, A. Badhwar, P. Orban, S. Belleville, H. Chertkow, A. Dagher, A. Hanganu, . Monchi, P. Rosa-Neto, A. Shmuel, S. Wang, J. Breitner, P. Bellec, and, "Consistent inter-protoce

11. A. Demertzi, G. Antonopoulos, L. Heine, H. U. Voss, J. S. Crone, C. de Los Angeles, M. A. Bahri, C. Di Perri, D. Charland-Verville, M. Kronbichler, E Trinka C Phillins, F. Gomez, L Thibanda, A. Soddu, N. D. Schiff, S. Whitfield-Gabrieli, and S. Laureys, "Intrinsic functional connectivity differentiates minimally conscious from unresponsive patients," Brain, 2015.

12. E. Bullmore and O. Sporns, "Complex brain networks: graph theoretical analysis of structural and functiona systems," Nat. Rev. Neurosci. 10, pp. 186-198, Mar 2009.

. M. Breakspear and C. J. Stam, "Dynamics of a neural system with a multiscale architecture," Philos. Tran R. Soc. Lond., B, Biol. Sci. 360, pp. 1051-1074, May 2005.

4. M. P. van den Heuvel and O. Sporns, "Rich-club organization of the human connectome," The Journal of Neuroscience 31(44), pp. 15775-15786, 2011.

15. K. J. Friston, "Functional and effective connectivity: a review," Brain Connect 1(1), pp. 13-36, 2011. 16. O. Sporns, "Networks analysis, complexity, and brain function," Complex. 8, pp. 56-60, Sept. 2002.

Deco, G. Tononi, M. Boly, and M. L. Kringelbach, "Rethinking segregation and integration: contribution f whole-brain modelling," Nat Rev Neurosci 16, pp. 430-439, July 2015.

18. C. Brummitt, D. R. M, and E. Leicht, "Suppressing cascades of load in interdependent networks," Proceed-

" "The comentomics of brain disorders," Nature Reviews Neuroscience 16, pp. 159-172, Feb. 2015. 
20. S. Fortunato, "Community detection in graphs," 486, pp. 75-174, Feb. 2010.

21. L. Djerou, N. Khelil, N. Dehimi, and M Batouche "Automatic multi-level thresholding segmentation based 2012

22. M. Girvan and M. E. J. Newman, "Community structure in social and biological networks," Proceedings of the National Academy of Sciences 99(12), pp. 7821-7826, 2002.

23. M. E. J. Newman, Fast algorithm for detecting community structure in networks, vol. 69, American Physical Society, Jun 2004

24. H. Trevor, T. Robert, and F. Jerome, The Elements of Statistical Learning: Data Mining, Inference, and Prediction. Second Edition, 2009.

25. C. Schnakers, S. Majerus, J. Giacino, A. Vanhaudenhuyse, M. A. Bruno, M. Boly, G. Moonen, P. Damas, B. Lambermont, M. Lamy, F. Damas, M. Ventura, and S. Laureys, "A French validation study of the Coma

26. B. e. a. Cecile, "Temporal and spatial independent component analysis for fmri data sets embedded in the 7.

S. Kindermann. A. J. Bell, and "Analysis of fmri data by blind separation into independent sotial compoct." Human Brain Mapping 6, pp. 160-188, 1998.

28. J. Kapur. P. Sahoo, and A. Wong, "A new method for gray-level picture thresholding using the entropy of the histogram," Computer Vision, Graphics, and Image Processing 29(3), pp. 273 - 285, 1985.

29. N. Otsu, "A threshold selection method from gray-level histograms," IEEE Trans. on Systems, Man, and Cybernetics 9(1), pp. 62-66, 1979.

. K. Deb, A. Pratap, S. Agarwal, and T. Meyarivan, "A fast elitist multi-objective genetic algorithm: Nsga-ii," IEEE Transactions on Evolutionary Computation 6, pp. 182-197, 2000.

1. R. Schulz, "Senior lecturer in bioinformatics and epigenomics department of medical and molecular genetics, king's college london." [Web; accedido el 06-11-2013].

32. R. Robinson, "Exploring the global workspace of consciousness," PLoS Biol 7, p. e1000066, 032009.

33. W. Seeley, V. Menon, A. Schatzberg, J. Keller, G. Glover, H. Kenna, A. Reiss, and M. Greicius, "Dissociable intrinsic connectivity networks for salience processing and executive control," The Journal of Neuroscience $27(9)$, pp. $2349-2556,2007$

34. O. Sporns, "Network attributes for segregation and integration in the human brain," Curr. Opin. NeuroD.J.

(E. P. Davis, C. A. Sandman, O. Sporns, B. F. O'Donnell, C. Buss, and W. P. Hetrick, "Children's intellectual ability is associated with structural network integrity," NeuroImage 124, Part A, pp. 550 556,2016

36. M. Pedersen, A. H. Omidvarnia, J. M. Walz, and G. D. Jackson, "Increased segregation of brain networks in focal epilepsy: An fmri graph theory finding," NeuroImage: Clinical 8, pp. 536- 542, 2015.

37. M. Y. Chan, D. C. Park, N. K. Savalia, S. E. Petersen, and G. S. Wig, "Decreased segregation of brain systems across the healthy adult lifespan," Proceedings of the National Academy of Sciences 111(46), pp. E4997-E5006, 2014 